\title{
Chimeric mRNA based COVID-19 vaccine elicits potent neutralizing antibodies and protection against Omicron and Delta
}

Qidong $\mathrm{Hu}^{1}{ }^{*}$, Ying $\mathrm{Zhao}^{1}{ }^{*}$, Namir Shaabani ${ }^{1 *}$, Xiaoxuan Lyu $^{1}$, Haotian Sun ${ }^{1}$, Vincent Cruz ${ }^{1}, \mathrm{Yi} \mathrm{Kao}^{1}$, Jia $\mathrm{Xu}^{1}$, Amber Fossier $^{1}$, Karen Stegman ${ }^{1}$, Zhihao Wang ${ }^{1}$, Zhenping Wang ${ }^{1}$, Yue Hu ${ }^{1}$, Yi Zheng ${ }^{1}$, Lilian Kyaw ${ }^{1}$, Cipriano Zuluaga ${ }^{1}$, Hua Wang ${ }^{1}$, Hong Pei ${ }^{1}$, Colin Powers ${ }^{1}$, Robert Allen ${ }^{1}$, Hui Xie ${ }^{1}$, Henry $\mathrm{Ji}^{1}$, Runqiang Chen ${ }^{1 \#}$

${ }^{1}$ Sorrento Therapeutics Inc., 4955 Directors Place, San Diego, CA, USA

*These authors contributed equally to this work as co-first authors.

${ }^{\#}$ Corresponding author. Email: rchen@sorrentotherapeutics.com (R.C.) 


\section{Abstract}

The emerging SARS-CoV-2 variants of concern (VOCs) exhibit enhanced transmission and immune escape, reducing the efficacy and effectiveness of the two FDA-approved mRNA vaccines currently in use. Here, we explored various strategies to develop mRNA vaccines that offer potentially safer and wider coverage of VOCs. We first introduced a furin cleavage mutation into the spike (S) protein of predominant VOCs, including Alpha (B.1.1.7), Beta (B.1.351), Gamma (P.1) and Delta (B.1.617.2), and confirmed the expression and cleavage deficiency of furin mutants. We then assessed the capacity of the resultant mRNAs to induce neutralizing antibodies (nAb) in mice following intramuscular administration. The initial mouse vaccination results showed that the individual VOC mRNAs induced the generation of neutralizing antibody in a VOC-specific manner. Moreover, we discovered that the antibodies produced from mice immunized with Beta-Furin and Washington (WA)-Furin mRNAs crossreacted with other VOCs. The broad spectrum of generated nAb was further confirmed when vaccinated mice were challenged with the respective live viruses. However, neither WA-Furin nor Beta-Furin mRNA elicited potent neutralizing activity against the omicron variant. Interestingly, in a mix-and-match booster experiment, omicron-Furin and WA-Furin mRNA elicited comparable protection against omicron. Finally, we tested the concept of bivalent vaccine by introducing the RBD of Delta strain into the intact $S$ antigen of Omicron. The chimeric mRNA induces potent and broadly acting nAb against Omicron and Delta, which paves the way to develop vaccine candidate to target emerging variants in the future.

Running title: Chimeric mRNA vaccine protects against Omicron and Delta 


\section{Introduction}

The global pandemic caused by the severe acute respiratory syndrome coronavirus 2 (SARS-CoV-2) has already claimed millions of lives since the outbreak in January 2020 (Adam, 2022). Numerous strategies have been employed to combat the spread of this infectious disease by public health agencies worldwide. As one of the most widely adopted approaches, vaccination has been proved to be a key tool to tackling COVID-19. To date, there are a total of ten COVID-19 vaccines granted for emergency use or fully approved globally (https://covid19.trackvaccines.org/agency/who/). Among the various types of vaccine platforms, the mRNA vaccine has been proved to be effective against COVID-related hospitalization and death (https://www.cdc.gov/coronavirus/2019-ncov/science/science-briefs/fullyvaccinated-people.html).

Despite these efforts, the pandemic continues to pose a threat to the public health due to the constant viral evolution and the consequential emergence of new SARS-CoV-2 variants of concern (VOCs) (Otto et al., 2021). Hence, there is an urgent need to develop more efficacious mRNA vaccines to fight against the current and potentially future VOCs.

The designated VOCs have caused multiple waves of widespread outbreak with unprecedented speed. Back in 2020, the first VOC Alpha (B.1.1.7) was detected in the United Kingdom, leading to more than 100,000 confirmed cases in the country. Following the emerging of Alpha variant, there were two other outbreaks originating from South Africa and Brazil, triggered by the Beta (B.1.351) and Gamma (P.1) variants, respectively (Adam, 2022; Liu et al., 2021; Otto et al., 2021). With additional mutation in spike protein, both VOCs were estimated to be $40 \%-80 \%$ more transmissible than the wildtype lineage. Furthermore, the Beta variant exhibits great immune escape and COVID-19 vaccination only showed 75\% effectiveness against infection (Abu-Raddad et al., 2021). In late 2020, a new variant B. 1.617 .2 (Delta) was identified and subsequentially contributed to a surge in cases in India and worldwide shortly afterwards. Besides the increased transmissibility, Delta variant has been shown to cause more severe disease and result in a poorer prognosis than previously reported VOCs (Farinholt et al., 2021; Mlcochova et al., 2021; Planas et al., 2021). To make things even worse, the effectiveness of existing mRNA vaccines against Delta has dropped considerably, from $~ 95 \%$ for the original WA strain to $76 \%$ (mRNA-1273) and 42\% (BNT162b2) (Puranik et al., 2021). By June 2021, global COVID-19-related deaths hit 5 million as Delta variant swept over 161 countries around the world.

More recently, WHO declared a novel VOC B.1.1.529, designated as Omicron, on $26^{\text {th }}$ Nov 2021, only two days after it was first reported to WHO by South Africa (Xu et al., 2022). Omicron quickly 
outcompeted the circulating Delta variant within weeks after landing in Europe and US, and has become the dominant $V O C$ around the globe. Scientists found that there was a significant reduction in Omicron virus neutralizing activity in sera obtained from individuals infected by pre-Omicron variants and populations vaccinated with immunogens based on early WA-1 virus (Cao et al., 2021; Lee et al., 2022; Zhao et al., 2021). A huge number of breakthrough infections have been reported even from individuals fully vaccinated with the $3^{\text {rd }}$ booster shot. Although still largely effective in preventing hospitalization and mortality, the two existing mRNA-based vaccines have shown declining capabilities in preventing infection by various VOCs, especially Omicron. A plausible explanation for the increasingly large gap in vaccine coverage is that both EUA-approved mRNA vaccines encode the $S$ protein of the original WA strain as the immunogen.

Compared to its ancestral strain, Omicron contains more than 30 additional mutations within the $S$ protein coding sequence, 15 of which reside in the receptor binding domain (RBD)(Wu et al., 2022). Capable of binding to the human ACE2 receptor, pioneering structural analysis demonstrated that RBD is the key domain for virus attachment and entry to human cells (Shang et al., 2020a; Shang et al., 2020b; Han et al., 2022; Lupala et al., 2022). Thus, the high rate of mutations in Omicron RBD may dramatically alter the interaction dynamics between the virus and host cell, which could, at least partially, explain the enhanced transmissibility and breakthrough cases (Liu et al., 2021; Zhao et al., 2021).

In the present study, we explored various strategies to develop mRNA vaccines that can provide broad and effective protection against predominant SARS-CoV-2 VOCs. We first screened five monovalent mRNA vaccines encoding the spike (S) protein of Alpha, Beta, Gamma, Delta, and the original Washington (WA) strain. All the vaccines harbor the mutation that abolishes the furin-mediated cleavage between S1 and S2 domains of S protein. It was found that Beta-Furin and WA-Furin mRNAs showed the most potent cross-neutralizing activity against other VOCs, with the exception of Omicron. Hence, we further constructed the mRNA vaccine encoding the $S$ protein of Omicron, which was shown in subsequent experiments to induce the strongest protection against Omicron. However, it did not elicit broad neutralizing capacity against other VOCs, such as Delta. To address the observed limitations of an Omicron-specific mRNA vaccine, we developed a chimeric mRNA by incorporating an extra RBD from Delta variant in the context of the $S$ protein of Omicron. The resultant chimeric mRNA vaccine elicited significantly higher cross-neutralization activity against Delta, while retaining efficacy against Omicron. Our findings thus provide insights into the development of the next generation mRNA vaccine with wider coverage of possible future VOCS of SARS-CoV-2, and the potential to provide vaccine coverage for 
bioRxiv preprint doi: https://doi.org/10.1101/2022.03.04.483032; this version posted March 7, 2022. The copyright holder for this preprint (which was not certified by peer review) is the author/funder. All rights reserved. No reuse allowed without permission.

respiratory pathogens outside of the family Coronaviridae. 


\section{Results}

Immunization with SARS-CoV-2 spike mRNA harboring furin cleavage mutation induces VOC-specific IgG production

Previously, we have designed a SARS-CoV-2 spike (S) mRNA vaccine that achieves high expression in mammalian cells (Francis et al., 2021). This mRNA vaccine encodes the $S$ protein from the Wuhan/Washington (WA) strain and encodes a polybasic furin cleavage site at the junction of S1 and S2 subunits. The feature could affect the stability of spike protein and reduce the pool of antigenic epitopes available to induce cellular and humoral immunity (Peacock et al., 2021). In addition, cleaved S1 was detected in the blood of immunized subjects receiving existing mRNA vaccine (Ogata et al., 2022), raising a significant question of whether this free moiety could represent a potential safety issue by mimicking full-length $S$ protein to activate ACE2, and thus trigger some side effects, including myocarditis in healthy subjects. Thus, to further optimize the mRNA vaccine and eliminate these potential safety concerns, the furin cleavage site between the S1 and S2 domains of the spike was mutated (Figure S1A) in the currently reported build of our mRNA vaccine candidates.

We then constructed two sets of mRNAs encoding the S protein of all the predominant SARS-CoV- 2 VOCs, one with the wild-type (WT) furin cleavage site, the other with mutated site. We examined and compared the expression of these VOC mRNAs, including WA, Alpha, Beta, Gamma and Delta in 293T cells. The flow cytometry results showed that removal of the furin cleavage site increased the surface expression of VOC S protein in transfected 293T cells (Figure S1B), and Western blot confirmed that the mutation abolished the furin cleavage and lowered the level of free S1 in the conditioned medium (Figure S1C, D). We thus hypothesized that the boosted expression of the furin mutant could result in stronger immunogenicity. To test the hypothesis, WA WT or furin mutant mRNAs were formulated with an in-house lipid nanoparticle (LNP), and six-week-old female BALB/c mice were intramuscularly injected with two doses of each LNP-mRNA separated by three weeks. ELISA on the sera collected 14 days after boost revealed that mRNA carrying the furin cleavage mutation elicited a higher average endpoint titer (EPT) of total binding antibody than its WT version (Figure S1E). We then performed the Plaque Reduction Neutralization Test (PRNT) using the same antisera to assess neutralizing antibody (nAb)(Nyiro et al., 2019; Schmidt et al., 1976). Consistent with ELISA data, PRNT results confirmed the superior neutralizing activity of sera from WA furin mutant-injected mice (Figure S1F) as compared to that measured in mice vaccinated with the WT version. 
Likewise, to investigate the ability of individual SARS-CoV-2 VOC mRNA vaccines to generate nAb, and to profile their coverage spectrum in vivo, we immunized six-week-old female BALB/c mice with LNPencapsulated furin-mutant VOC mRNAs and compared the performance of individual mRNAs in vaccinated mice. The EPT of total binding antibodies was first measured by ELISA using the sera day 14 post two-dose injection. As expected, each mRNA induced the strongest antibody response against the corresponding VOC S protein, with the exception of Gamma (Figure 1A). Intriguingly, some VOC mRNA vaccinations led to the production of antibodies capable of binding to a breadth of VOC S proteins, especially Beta-Furin. To further analyse the neutralizing capability of individual sera, PRNT was performed where VeroE6 cells were exposed to the live virus of five VOCs in the absence or presence of diluted serum collected from the immunized mice (Figure 1B). In keeping with the ELISA results, the experiment showed that the individual monovalent mRNA vaccine generally displayed variant-specific protection activity. For example, WA-Furin mRNA induced the highest neutralizing activity against the WA-1 virus. Again it was observed that the serum from the Beta-Furin mRNA injected cohort displayed robust and broad protection against all VOCs tested. Particularly, the Beta-Furin mRNA provided a much stronger protection against the highly contagious Delta variant than was elicited by vaccination with the WA-Furin mRNA. The data indicates that whereas the VOC-specific strategy can provide strain-specific protection, some VOC-based mRNA vaccines have relatively enhanced potential to trigger a broad and potent immune response to the genetically divergent set of existing SARS-CoV-2 variants.

\section{Immunization with SARS-CoV-2 spike vaccines carrying furin cleavage mutation produces robust protection in vivo}

The K18-hACE2 transgenic model has been extensively utilized to evaluate the vaccine efficacy and effectiveness in preventing COVID-19 in the preclinical setting (Arce and Costoya, 2021; Dong et al., 2021; Radvak et al., 2021; Winkler et al., 2020). Two key metrics to determine the severity of pathogenesis are the virus titer in the lung tissue and body weight loss following virus infection. To investigate the protection capacity of our furin-mutant mRNA vaccines, K18-hACE2 mice were first intramuscularly administrated with $5 \mu \mathrm{g}$ of WA-Furin or Beta-Furin mRNA twice with 3-week interval. Five weeks post full vaccination, the animals were challenged with $1 \times 10^{5}$ half-maximal tissue culture infectious dose (TCID50) of the WA, Beta or Lambda variants (Figure 2A). Then the virus replication in the lung was quantified to determine the effect of vaccination. The average virus titer was approximated to $1 \times 10^{6}, 2.1 \times 10^{5}$, and $4.5 \times 10^{6} \mathrm{TCID50/g}$ for WA, Beta and Lambda strains, respectively in the control 
group injected with PBS. On the other hand, immunization with either WA-Furin or Beta-Furin mRNA almost completely inhibited the replication of virus in the lungs, with virus titers falling below the limit of detection (Figure 2B). As expected, animals treated with PBS exhibited dramatic weight loss in all challenge settings, regardless of the virus strain. The average body weight in the PBS controls declined to $82 \%, 78 \%$ and $81 \%$ on day 5 post infection with WA, Beta and Lambda strains, respectively. In contrast, none of the mice immunized with Beta-Furin or WA-Furin vaccines showed any sign of weight loss, and some animals in these treatment groups gained weight after infection up to 5 days (Figure 2C). In addition, both furin-mutant mRNAs gave effective protection against the Lambda variant although the corresponding spike mRNA was not included among the immunogens, suggesting broad protection capacity of some VOC mRNAs.

\section{Omicron variant undermines the effectiveness of the past VOC based vaccines}

Recently, a new SARS-CoV-2 variant B.1.1.529 (Omicron), was identified in South Africa and declared as VOC by WHO due to its rapid rise in global prevalence. Due in large part to an unusually large number of previously unreported RBD mutations as compared to other VOCs, Omicron exhibited the most significant escape from the serum of both fully vaccinated subjects and convalescents (Planas et al., 2022). To investigate whether our optimized mRNA vaccines could offer effective protection against Omicron, VSV pseudotyped viruses were used to determine the neutralization potency of sera collected from vaccinated animals (Figure 3A). In agreement with other studies (Muik et al., 2022), we found that the mean neutralization titer (IC50) of Omicron was only 318 for the sera collected at day 14 after boost with WA-Furin mRNA, which represents 7.7-fold decrease of neutralization, as compared to that recorded against Delta variant-based pseudotyped viruses (data not shown). A similar decrease in the Omicron protection capacity of the sera was observed for mRNA vaccine candidates derived from other VOCs (Figure 3A). Surprisingly, even the Beta-Furin mRNA, which has been shown to provide the broadest immune response against all major VOCs (Figure 1), failed to induce sufficient protection against Omicron, prompting us to explore further immunogen designs to overcome this liability.

To establish the potential of a variant-matched monovalent vaccine to provide protective immunity against Omicron virus, we replaced the coding sequence of the original mRNA vaccines with Omicron spike retaining the furin cleavage mutation. More than $85 \%$ of the Omicron-Furin mRNA transfected 293T cells, but not the mock control, could be detected by flow cytometry with Fc-tagged ACE2 recombinant protein (Figure S2). We then performed in vivo studies to assess the immunogenicity and efficacy of the Omicron-specific mRNA vaccine. BALB/c mice were immunized intramuscularly with $5 \mu \mathrm{g}$ 
Omicron-LNP mRNA or Beta-LNP mRNA two times at a three-week interval. Serum was collected two weeks post boost and subjected to ELISA assay to measure Omicron-specific binding antibodies. Using the recombinant Omicron spike protein as the coating antigen, high titers of binding antibodies were observed in the sera of Omicron mRNA-injected mice (Figure 3B). The protective capability of Omicronspecific mRNA vaccine was further evaluated in PRNT. We confirmed that the sera collected from Omicron-Furin-immunized animals offered superior protection against the infection of Omicron strain (Figure 3C), demonstrating this newly designed mRNA vaccine could induce potent production of Omicron-specific nAbs in vivo.

As the Omicron strain led to increasing number of breakthrough cases even in people already receiving three-dose vaccination, we wanted to investigate whether using Omicron-Furin mRNA solely as a boosting immunogen could provide enhanced protection. Hence, we set up an in vivo challenge study to simulate the real-world scenario (Figure 3D). Two doses of WA-Furin mRNA were administrated into K18-ACE2 transgenic mice as described previously. Five weeks after the second dose, the animals received the booster shot of either $5 \mu \mathrm{g}$ WA-Furin mRNA or Omicron-Furin mRNA. After another five weeks, the animals were challenged with live Omicron strain before lung virus loads were quantified. Surprisingly, while the control group displayed high viral titer, up to $5 \times 10^{\wedge} 5 \mathrm{PFU}$ per lung, vaccinated mice showed no detectable viral replication in the lung, suggesting that both WA-1 and Omicron-based booster mRNAs provided substantial and similar protection against Omicron.

\section{Chimeric RBD-based mRNA vaccine elicits broad protection against Omicron and Delta}

With the new omicron mRNA vaccine, we sought to determine whether it could provide sufficient crossreactive immunity against other VOCs. The ELISA and pseudovirus assays indicated that the OmicronFurin mRNA vaccine elicited only limited immunity against VOCs, especially Delta (data not shown). Hence, in an effort to generate a vaccine with broad cross-reactivity against other VOCs, we constructed a chimeric VOC immunogen by inserting the RBD domain of the Delta variant directly upstream of the Omicron RBD within the Omicron spike backbone (Figure 4A). We first confirmed that the translation product of the chimeric mRNA could still bind to its natural receptor, ACE2 by flow cytometry (Figure S2). To evaluate the immunogenicity and efficacy of this chimeric design, mice were immunized twice with LNP-formulated mRNA as described above. The sera were collected two weeks following the second dose and then analyzed for the titers of binding antibodies and nAbs against various VOCs. The ELISA results showed that the chimeric Delta RBD-Omicron mRNA outperformed the original Omicron mRNA in the generation of binding antibodies against WA, Beta and Gamma variants (Figure 4A). 
Surprisingly, although a moderately higher titer against Delta was seen with the chimeric mRNA vaccination, it did not reach statistical significance. As the readout of ELISA assay is not a direct indicator of neutralizing capability, we used the same serum panel to further quantify the nAb titer against Delta and two Omicron pseudoviruses (Figure 4B). The IC50 of neutralization showed that compared to Omicron-Furin mRNA, the Delta RBD-Omicron immunization induced similar neutralization activity towards the original and R346K Omicron sub-variant. The absolute IC50 was 5595 with Omicron-Furin and 4945 with Delta RBD-Omicron against the ancestral omicron strain, while the corresponding readout jumped to 8485 and 7589 against Omicron R346K, respectively. Notably, there was a significant increase in the $\mathrm{nAb}$ titer against the Delta variant when mice were immunized with the chimeric Delta RBD-Omicron mRNA as compared to mice immunized with the Omicron-Furin mRNA. Taken together, this chimeric design offers a powerful strategy to develop mRNA vaccines with broad protection capacity against COVID-19 and other infectious diseases. 


\section{Discussion}

Prophylactic nucleic acid vaccines can deliver the nucleotide sequence that codes for virus-derived but nonpathogenic proteins into host cells, thus mimicking a native infection to elicit an immune response. Unlike DNA, mRNA vaccines eradicate the need for nucleic acid to enter the nucleus to achieve expression, and they are less likely to be integrated into the host genome. There are currently two mRNA-based SARS-CoV-2 vaccines authorized by FDA and widely disseminated. These vaccines encode for the $S$ protein, the major surface protein on the coronavirus virion responsible for anchoring onto target cells, and thus the predominant virus-encoded target for nAb elicited by natural infection. Although clinical trials and real-world data have affirmed the safety and effectiveness of these FDAauthorized COVID-19 vaccines, more and more breakthrough infections have been reported for predominant VOCs. For example, the effectiveness of BNT162b2 against Delta-caused infection plummeted to $42 \%$, as compared to $95 \%$ against the ancestral WA strain (Puranik et al., 2021), highlighting the need of developing vaccines that can offer wide protection against persistently emerging VOCs.

As each VOC possesses its unique set of mutations in the $S$ protein, this will invariably result in distinct pools of epitopes being presented to lymphocytes by antigen presenting cells (APCs). Hence, we first evaluated the protection conferred following vaccination with mRNA vaccines encoding the $S$ proteins of VOCs that emerged prior to Omicron. Our in vitro and in vivo data clearly demonstrated the premise that the strongest immunity against individual VOCs can be achieved by vaccination with variant-specific mRNA. For example, immunization with Delta S mRNA provided the best protection against Delta, but not to other VOCs (Figure 1). We also noticed that WA and Beta mRNA, especially the latter, provided the widest breadth of coverage for other VOCs. This could be explained by some mutations within Beta S protein, especially in the N-terminal and RBD. These mutations are also present in other VOCs, for example, L18, K417, E484, N501, D614, some of which are known to cause great immune escape (Grabowski et al., 2021; Winger and Caspari, 2021). Interestingly, Beta S mRNA has also been selected by Moderna to test in phase II either as a monovalent antigen or by mixing with mRNA-1273 to tackle the emerging VOCs (Pajon et al., 2022; Waltz, 2021).

With the emergence and rampage of Omicron during the course of our candidate vaccine development, we further tested the effectiveness of non-Omicron VOC mRNA vaccines against this new variant. Not surprisingly, none of these candidates induced strong immunity when challenged with Omicron (Figure 3A). This could be explained by the more than 30 mutations in the $S$ protein of Omicron, a mutational 
burden which far exceeded that of preceding VOCs. This observation is consistent with the complete escape of Omicron from most of EUA-approved neutralizing antibody and convalescent sera therapies (Liu et al., 2022). To recapitulate our previous observation that VOC-matched vaccine induces the strongest $n A b$ response in a VOC-dependent manner, we constructed a new mRNA vaccine encoding the $S$ protein of Omicron and it indeed provided superior protection against this new VOC (Figure 3B, C). The notion was reciprocally confirmed by the observation that Omicron mRNA did not induce strong immunity against other VOCs.

As the world is witnessing an astounding number of Omicron-related breakthrough cases even in people have received a third dose of currently available mRNA vaccines, a serious concern has been raised of whether a booster is adequate to stop its spread. Hence, we compared Omicron-specific booster to WAspecific booster to explore any additive protection. However, our data argued that the heterologous booster scheme is not superior to boosting with the ancestral WA mRNA in terms of establishing immunity against Omicron (Figure 3D). Similar conclusion was drawn by a research group from NIH, when they challenged mRNA-1273-vaccinated macaques with live omicron virus (Gagne et al., 2022). A plausible explanation is although Omicron S protein has $~ 35$ mutations, it still exhibits $97 \%$ similarity to the ancestral WA strain in the amino acid sequence. As a result, most of the epitopes presented to lymphocytes could remain the same, and the consequence of antigenic drift contributed by those 35 mutations could be masked by the surge of nAbs generated after the third dose. Hence, individuals with prior immunity from vaccination may not necessarily benefit from a change in vaccinating antigens. Moreover, considering the cost and time needed to put variant specific mRNA vaccine to practical use, the homologous boost scheme remains a scientifically proven and economically feasible option at hand in the fight against COVID-19.

Nonetheless, the concept of universal vaccine is still very appealing for at least two reasons. One is the virus could keep accumulating more mutations to eventually nullify the effectiveness of existing mRNA vaccines. The other is the idea can be applied to establish immunity against viruses causing different diseases. For example, dimeric RBDs have been ligated in tandem to target MERS, SARS and COVID-19 (Dai et al., 2020). In comparison, our chimeric design included the incorporation of the RBD of Delta variant in the Omicron spike mRNA which offers a larger pool of epitopes. Remarkably, we found that the resultant $\mathrm{mRNA}$ restored the strong protection against Delta infection while retaining the effective immunity against Omicron (Figure 4). Similar strategies have also been explored to target the Sarbecovirus subgenus with a single-molecule antigen (Martinez et al., 2021), suggesting broad 
applications of the chimeric vaccine design to deliver effective protection against a wide panel of diseases.

Another unique feature of our in-house mRNA vaccine is the mutation of furin cleavage site between the S1 and S2 domains of S. This cleavage is believed to have emerged during viral transmission from its zoonotic host to humans and is one of the key attributes to explain the high transmissibility of SARSCoV-2 in humans (Whittaker, 2021). The mutation is mainly to address the concern that circulating S1 was detected in the plasma of vaccinated subjects (Ogata et al., 2021). Although the clinical consequence of the free S1 moiety has not been full established, studies have suggested that S1 can be taken up by many critical organs, such as liver, kidney, spleen, and even cross the blood-brain barrier to gain access to the brain (Rhea et al., 2021). As S1 contains the intact RBD, it could still bind to ACE2 and trigger the downstream signaling events that may lead to inflammation and lung damage (Letarov et al., 2021; Suzuki and Gychka, 2021). Hence, we mutated the furin cleavage site and confirmed the abrogation of S1 liberation in both the cell lysates and conditioned medium. Although a similar design was adopted in the recombinant protein-based NVX-CoV2373 by Novovax, it is not present in the two mRNA-based Covid-19 vaccines authorized by FDA. Another potential benefit of furin cleavage mutation is by retaining the full-length S protein within the cell and on the cell surface (Figure S1B), a larger pool of antigens could become available for presentation to induce the adaptive immunity. Indeed, S protein with furin cleavage mutation even binds with higher affinity to ACE2 (Laczkó et al., 2020).

Taken together, our in-house designed mRNA vaccine represents a potentially safer alternative to existing products in use and could induce stronger adaptive immunity against prevalent VOCs, including Omicron and Delta. Our chimeric design will also facilitate the development of next-generation vaccines that achieve the balance between effectiveness and coverage, not only for the variants of SARS-CoV- 2 but also for other viruses. 


\section{Materials and Methods}

\section{In vitro transcription and purification of RNA}

To generate the template for RNA synthesis, the sequences of the SARS-Cov-2 Spike protein of VOC were codon optimized and cloned into pVAX1-based backbone which features 5?-UTR, 30?-UTR and PolyA tail. To increase the protein stability, 2P mutations at positions 986-987 were introduced. The plasmid DNA was produced in bacteria, purified and linearized by a single-site restriction enzyme digestion. The template DNA was purified, spectrophotometrically quantified, and in vitro transcribed by T7 RNA polymerase (Cat: M0251, NEB) in the presence of a trinucleotide cap1 analogue,

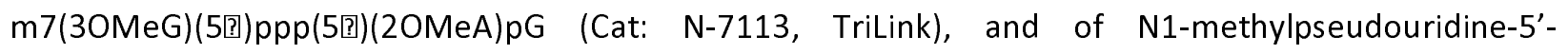
triphosphate (Cat: N-1081, TriLink) in place of uridine-5'-triphosphate (UTP). After the reaction, DNase I (Cat: M0303, NEB) was added to remove the template DNA and the mRNA was purified by LiCl precipitation (Cat: AM9480, ThermoFisher).

\section{mRNA formulation}

LNPs were prepared by microfluidic mixing a buffered solution of mRNA with an ethanol solution of lipids [distearoylphosphatidylcholine (DSPC), cholesterol, 1,2-Dimyristoyl-rac-glycero-3methoxypolyethylene glycol-2000 (DMG-PEG2000), and ionizable lipid. The LNPs were concentrated by dialysis against an aqueous buffer system, following a $0.2 \mu \mathrm{m}$ sterile filtration. The LNPs were tested for mRNA concentration, encapsulation efficiency, particle size, $\mathrm{pH}$, and osmolality.

\section{In vitro mRNA expression}

Monocytes were isolated from PBMCs and differentiated into DCs in presence of GM-CSF (Cat: 300-03, Peprotech) and IL-4 (Cat: 200-04, Peprotech). Between day 6-day 8, cells were transfected with mRNA by the NeonTM electroporation transfection system (Cat: MPK5000, ThermoFisher). 24 hours posttransfection, the cells were collected and subjected to flow cytometry as described below to check the expression of spike. With $293 \mathrm{~T}$ adherent cells, mRNA (2.5ug) of WT vs Mutant from five variants (Washington, Alpha, Beta, Gamma and Delta) were transfected with Lipofectamine ${ }^{\mathrm{TM}}$ MessengerMAX $^{\mathrm{TM}}$ Transfection Reagent ( $2 \mathrm{ul}$ ) and cultured for 72 hours at $370 \mathrm{C}$ using $293 \mathrm{~T}$ adherent cells with $0.5 \mathrm{ml}$ of DMEM media with $10 \%$ FBS in each well of a 24 -well cell culture-treated plate. Transfected cells from each well were dislodged with $400 \mathrm{ul}$ of TrypLE at 72 hours and neutralized with its own media. Cell pellets were collected after spinning down at 550g for 2 minutes by removing supernatant for each well. 


\section{Flow cytometry}

The collected cell pellets were washed with 250ul of FACS buffer (DPBS + 0.5\% BSA) in 96 wells treated plate followed by 30-minute incubation with the in-house STI-2020 (for DCS) or 10A3 (for 293T cells) primary antibody (1:1000) to detect SARS Cov-2 spike. 200ul FACS buffer was used to wash the cells twice with the same speed and time after 30-minute incubation, followed by rat anti-human Fc antibody conjugated to APC (Cat: 410712, BioLegend; 1:100 dilution) for 15 min on ice in the dark for secondary detection. The cells were spun down and the pellets were washed twice with the same speed and time of centrifugation using 200ul FACS buffer and resuspended in 200ul FACS buffer. The fluorescent intensity of positive cells within the gated population was detected by the Attune NxT Flow Cytometer (ThermoFisher) using 100 ul of acquisition volume setting.

\section{SARS-CoV-2 Virus}

SARS-COV-2 viruses were obtained from BEl resources (Washington strain NR-52281; Alpha variant NR54000; Beta Variant NR-54009; Gamma variant NR-54982; Delta variant NR- 55611 or NR-55672; Lambda variant NR- 55654 and Omicron NR-56461). VeroE6 monolayers were infected at an MOI of 0.01 in $5 \mathrm{ml}$ virus infection media (DMEM $+2 \% \mathrm{FCS}+1 \mathrm{X}$ Pen/Strep). Tissue culture flasks were incubated at $36^{\circ} \mathrm{C}$ and slowly shaken every 15 minutes for a 90-minute period. Cell growth media $(35 \mathrm{~mL})$ was added to each flask and infected cultures were incubated at $36^{\circ} \mathrm{C} / 5 \% \mathrm{CO} 2$ for 48 hours. Media was then harvested and clarified to remove large cellular debris by room temperature centrifugation at $3000 \mathrm{rpm}$.

\section{Animals and in vivo studies}

7-week-old $\mathrm{BALB} / \mathrm{cJ}$ female mice were purchased from the Jackson Laboratory. All protocols were approved by the Institutional Animal Care and Use Committee (IACUC). mRNA formulations were diluted in $50 \mathrm{uL}$ of $1 \mathrm{X}$ PBS, and mice were inoculated IM into the same hind leg for both prime and boost. There was 3 weeks interval between prime and boost. Two weeks after boost, mice blood was collected from retro-orbital for ELISA and pseudovirus neutralization assay.

\section{ELISA}

Ni-NTA HisSorb plates (Qiagen) were coated with 50ng/well of S1 proteins (all from Sino Biological, Cat: 40591-V08H, 40589-V08B6, 40589-V08B7, 40589-V08B8, 40589-V08B16) in 1 X PBS at $4^{\circ} \mathrm{C}$ overnight. To block the plate, Blocker Casein (Cat: 37528 Thermo) was used for 1 hour at room temperature (RT). After standard washes and blocks, plates were incubated with serial dilutions of sera for 1 hour at RT. 
Following washes, goat anti-mouse IgG (H+L)-HRP conjugate (Cat: \#1721011, Bio-Rad) were used as secondary Abs, and Pierce TMB substrate kit (Cat: 34021, Thermo Fisher) was used as the substrate. The absorbance was measured at 450 nanometers using a BioTek Cytaktion 5 plate reader. Endpoint tiers were calculated as the dilution that emitted an optical density exceeding $4 \mathrm{X}$ background (secondary Ab alone).

\section{Pseudovirus neutralization assay}

SARS-CoV-2 Spike pseudotyped $\triangle$ G-VSV-luciferase was generated by nucleofection of BHK cells (maintained in DMEM/F12 with 10\%FBS and 5\%TPB) with Spike-expressing plasmid followed by transduction with G-pseudotyped $\Delta G$-luciferase (G* ${ }^{*}$ G-luciferase) rVSV (Kerafast) 18-24 hours later. The supernatant containing pseudovirus was collected following 24 hours and stored at $-80^{\circ} \mathrm{C}$. Pseudovirus was normalized for luciferase expression using $G^{*} \Delta G$-luciferase VSV of known titer as the standard. For neutralization testing, HEK-Blue 293 hACE2-TMPRSS2 cells (Invivogen; maintained in DMEM with 10\% FBS) were plated to white-walled 96 -well plates at 40,000 cells/well and incubated at $37^{\circ} \mathrm{C} / 5 \% \mathrm{CO}$. The next day, SARS-CoV-2 Spike pseudotyped $\triangle$ G-VSV-luciferase was incubated with a dilution series of mouse serum (dilutions as indicated) and anti-VSV-G (Kerafast; $1 \mu \mathrm{g} / \mathrm{mL}$ ) antibody for 30 minutes at room temperature and added to the HEK-Blue 293 hACE2-TMPRSS2 cells. Transduced cells were incubated for 24 hours at $37^{\circ} \mathrm{C} / 5 \% \mathrm{CO} 2$ and luminescence measured by addition of $40 \mu \mathrm{l}$ of ONE-Glo reagent (Promega) with detection using a Tecan Spark plate reader. The percent inhibition was calculated using the formula 1-([luminescence of serum treated sample]/[average luminescence of untreated samples] x 100. The average of quadruplicate samples was included in the analyses.

\section{Plaque Reduction Neutralization Test (PRNT)}

Simian VeroE6 cells were plated at $18 \times 103$ cells/well in a flat bottom 96-well plate in a volume of 200 $\mu \mathrm{l} /$ well. After 24 hours, a serial dilution of seropositive blood serum is prepared in a $100 \mu \mathrm{l} /$ well at twice the final concentration desired and live virus was added at 1,000 PFU/100 $\mu$ l of SARS-CoV-2 and subsequently incubated for 1 hour at $37^{\circ} \mathrm{C}$ in a total volume of $200 \mu \mathrm{l} /$ well. Cell culture media was removed from cells and sera/virus premix was added to VeroE6 cells at $100 \mu \mathrm{l} /$ well and incubated for 1 hour at $37^{\circ} \mathrm{C}$. After incubation, $100 \mu \mathrm{l}$ of "overlay" (1:1 of $2 \%$ methylcellulose (Sigma) and culture media) is added to each well and incubation commenced for 3 days at $37^{\circ} \mathrm{C}$. Plaque staining using Crystal Violet (Sigma) was performed upon 30 min of fixing the cells with 4\% paraformaldehyde (Sigma) diluted in PBS. Plaques were assessed using a light microscope (Keyence). 


\section{Challenge study}

K18-hACE2 transgenic mice were purchased from Jackson laboratory and maintained in pathogen-free conditions and handling conforms to the requirements of the National Institutes of Health and the Scripps Research Institute Animal Research Committee. 8-12 weeks old mice were injected with the indicated administration technique under isoflurane anesthesia in the right hind flank area for IM injections. Mice were infected intranasally with 10000 PFU of SARS-CoV-2 in total volume $50 \mu \mathrm{L}$.

\section{Plaque assay}

VeroE6 cells were plated at $3 \times 10 \mathrm{e} 5$ cells/well in 24 well plates in volume $400 \mu \mathrm{l} /$ well. After $24 \mathrm{~h}$. medium is removed, and serial dilution of homogenized lungs were added to Vero cells and subsequently incubated for $1 \mathrm{~h}$ at $37^{\circ} \mathrm{C}$. After incubation, an overlay (1:1 of $2 \%$ methylcellulose (Sigma) and culture media) is added to each well and incubation commenced for $3 \mathrm{~d}$ at $37^{\circ} \mathrm{C}$. Plaque staining was performed using Crystal Violet as mentioned above.

\section{Statistics}

Statistical significance of differences between experimental groups was determined with Prism software (Graphpad). All data are expressed as standard error mean (SEM). ${ }^{* * * * P}<0.0001,{ }^{* * *} \mathrm{P}<0.001,{ }^{* *} \mathrm{P}<$ 0.01 , and ${ }^{*} \mathrm{P}<0.05$ by unpaired two-tailed $t$ tests or one- or two- way analysis of variance (ANOVA).

\section{Author Contributions}

R.C., H.J., Q.H., Y.Z., N.S., X.L. conceptualized and designed experiments. Q.H., Y.Z., N.S., X.L., H.S., V.C., Y.K., J.X., A.F., K.S., Z.W., Z.W., Y.H., Y.Z., L.K., C.Z., H.W. performed experiments. Q.H., Y.Z., N.S., X.L., H.S., H.P., C.P., R.A., H.X., R.C. analyzed and interpreted the data. Q.H., Y.Z., N.S., X.L. and R.C. wrote the paper.

\section{Competing interests}

Sorrento authors own options and/or stock of the company. This work has been described in one or more provisional patent applications. H.J. is an officer at Sorrento Therapeutics, Inc.

\section{Acknowledgments}


We thank Lisa Kerwin, Nancy Du and Yanliang Zhang for providing reagents; Avery Coyle and Charlotte Sadaka for helpful discussions; Brian Sun, Mike Ruse, Jr., Elizabeth Orr, Gali SteinbergTatman and Maggy Smith for patent applications.

\section{Reference}

Abu-Raddad, L.J., Chemaitelly, H., and Butt, A.A. (2021). Effectiveness of the BNT162b2 Covid-19 Vaccine against the B.1.1.7 and B.1.351 Variants. N. Engl. J. Med. 385, 187-189.

Adam, D. (2022). The pandemic's true death toll: millions more than official counts. Nature 601, 312315.

Arce, V.M., and Costoya, J.A. (2021). SARS-CoV-2 infection in K18-ACE2 transgenic mice replicates human pulmonary disease in COVID-19. Cell. Mol. Immunol. 18, 513-514.

Cao, Y., Wang, J., Jian, F., Xiao, T., Song, W., Yisimayi, A., Huang, W., Li, Q., Wang, P., An, R., et al. (2021). Omicron escapes the majority of existing SARS-CoV-2 neutralizing antibodies. 2021.12.07.470392.

Dai, L., Zheng, T., Xu, K., Han, Y., Xu, L., Huang, E., An, Y., Cheng, Y., Li, S., Liu, M., et al. (2020). A Universal Design of Betacoronavirus Vaccines against COVID-19, MERS, and SARS. Cell 182, 722-733.e11.

Dong, W., Mead, H., Tian, L., Park, J.-G., Garcia, J.I., Jaramillo, S., Barr, T., Kollath, D.S., Coyne, V.K., Stone, N.E., et al. (2021). The K18-Human ACE2 Transgenic Mouse Model Recapitulates Non-severe and Severe COVID-19 in Response to an Infectious Dose of the SARS-CoV-2 Virus. J. Virol.

Farinholt, T., Doddapaneni, H., Qin, X., Menon, V., Meng, Q., Metcalf, G., Chao, H., Gingras, M.-C., Farinholt, P., Agrawal, C., et al. (2021). Transmission event of SARS-CoV-2 Delta variant reveals multiple vaccine breakthrough infections. MedRxiv 2021.06.28.21258780.

Francis, D. Directing an mRNA-LNP vaccine toward lymph nodes improves humoral and cellular immunity against SARS-CoV-2. 23.

Francis, D.M., Chen, R., Khorsandzadeh, S., Hu, Q., Lyu, X., Wang, H., Lim, W., Sun, H., Xie, H., Shaabani, N., et al. (2021). Directing an mRNA-LNP vaccine toward lymph nodes improves humoral and cellular immunity against SARS-CoV-2. 2021.08.25.457699.

Frederic Grabowski et al., SARS-CoV-2 Variant of Concern 202012/01 Has about Twofold Replicative Advantage and Acquires Concerning Mutations. Viruses. 2021 Mar 1;13(3):392.

Gagne, M., Moliva, J.I., Foulds, K.E., Andrew, S.F., Flynn, B.J., Werner, A.P., Wagner, D.A., Teng, I.-T., Lin, B.C., Moore, C., et al. (2022). mRNA-1273 or mRNA-Omicron boost in vaccinated macaques elicits comparable B cell expansion, neutralizing antibodies and protection against Omicron. 2022.02.03.479037.

Grabowski F, Preibisch G, Giziński S, Kochańczyk M, Lipniacki T. SARS-CoV-2 Variant of Concern 202012/01 Has about Twofold Replicative Advantage and Acquires Concerning Mutations. Viruses. 2021 Mar 1;13(3):392. 
Han, P., Li, L., Liu, S., Wang, Q., Zhang, D., Xu, Z., Han, P., Li, X., Peng, Q., Su, C., et al. (2022). Receptor binding and complex structures of human ACE2 to spike RBD from omicron and delta SARS-CoV-2. Cell $185,630-640 . e 10$.

Laczkó, D., Hogan, M.J., Toulmin, S.A., Hicks, P., Lederer, K., Gaudette, B.T., Castaño, D., Amanat, F., Muramatsu, H., Oguin, T.H., et al. (2020). A Single Immunization with Nucleoside-Modified mRNA Vaccines Elicits Strong Cellular and Humoral Immune Responses against SARS-CoV-2 in Mice. Immunity 53, 724-732.e7.

Lee, I.-J., Sun, C.-P., Wu, P.-Y., Lan, Y.-H., Wang, I.-H., Liu, W.-C., Tseng, S.-C., Tsung, S.-I., Chou, Y.-C., Kumari, M., et al. (2022). Omicron-specific mRNA vaccine induced potent neutralizing antibody against Omicron but not other SARS-CoV-2 variants. 2022.01.31.478406.

Letarov, A.V., Babenko, V.V., and Kulikov, E.E. (2021). Free SARS-CoV-2 Spike Protein S1 Particles May Play a Role in the Pathogenesis of COVID-19 Infection. Biochem. Biokhimiia 86, 257-261.

Liu, L., Iketani, S., Guo, Y., Chan, J.F.-W., Wang, M., Liu, L., Luo, Y., Chu, H., Huang, Y., Nair, M.S., et al. (2022). Striking antibody evasion manifested by the Omicron variant of SARS-CoV-2. Nature 602, 676681.

Liu, Z., VanBlargan, L.A., Bloyet, L.-M., Rothlauf, P.W., Chen, R.E., Stumpf, S., Zhao, H., Errico, J.M., Theel, E.S., Liebeskind, M.J., et al. (2021). Identification of SARS-CoV-2 spike mutations that attenuate monoclonal and serum antibody neutralization. Cell Host Microbe 29, 477-488.e4.

Lupala, C.S., Ye, Y., Chen, H., Su, X.-D., and Liu, H. (2022). Mutations on RBD of SARS-CoV-2 Omicron variant result in stronger binding to human ACE2 receptor. Biochem. Biophys. Res. Commun. 590, 3441.

Martinez, D.R., Schäfer, A., Leist, S.R., De la Cruz, G., West, A., Atochina-Vasserman, E.N., Lindesmith, L.C., Pardi, N., Parks, R., Barr, M., et al. (2021). Chimeric spike mRNA vaccines protect against Sarbecovirus challenge in mice. Science 373, 991-998.

Mlcochova, P., Kemp, S.A., Dhar, M.S., Papa, G., Meng, B., Ferreira, I.A.T.M., Datir, R., Collier, D.A., Albecka, A., Singh, S., et al. (2021). SARS-CoV-2 B.1.617.2 Delta variant replication and immune evasion. Nature 599, 114-119.

Muik, A., Lui, B.G., Wallisch, A.-K., Bacher, M., Mühl, J., Reinholz, J., Ozhelvaci, O., Beckmann, N., Güimil Garcia, R. de la C., Poran, A., et al. (2022). Neutralization of SARS-CoV-2 Omicron by BNT162b2 mRNA vaccine-elicited human sera. Science $375,678-680$.

Nyiro, J.U., Kiyuka, P.K., Mutunga, M.N., Sande, C.J., Munywoki, P.K., Scott, J.A.G., and Nokes, D.J. (2019). Agreement between ELISA and plaque reduction neutralisation assay in Detection of respiratory syncytial virus specific antibodies in a birth Cohort from Kilifi, coastal Kenya. Wellcome Open Res. 4, 33.

Ogata, A.F., Cheng, C.-A., Desjardins, M., Senussi, Y., Sherman, A.C., Powell, M., Novack, L., Von, S., Li, X., Baden, L.R., et al. (2021). Circulating SARS-CoV-2 Vaccine Antigen Detected in the Plasma of mRNA-1273 Vaccine Recipients. Clin. Infect. Dis. Off. Publ. Infect. Dis. Soc. Am. ciab465. 
Ogata, A.F., Cheng, C.-A., Desjardins, M., Senussi, Y., Sherman, A.C., Powell, M., Novack, L., Von, S., Li, X., Baden, L.R., et al. (2022). Circulating Severe Acute Respiratory Syndrome Coronavirus 2 (SARS-CoV-2) Vaccine Antigen Detected in the Plasma of mRNA-1273 Vaccine Recipients. Clin. Infect. Dis. 74, 715-718.

Otto, S.P., Day, T., Arino, J., Colijn, C., Dushoff, J., Li, M., Mechai, S., Van Domselaar, G., Wu, J., Earn, D.J.D., et al. (2021). The origins and potential future of SARS-CoV-2 variants of concern in the evolving COVID-19 pandemic. Curr. Biol. 31, R918-R929.

Pajon, R., Doria-Rose, N.A., Shen, X., Schmidt, S.D., O’Dell, S., McDanal, C., Feng, W., Tong, J., Eaton, A., Maglinao, M., et al. (2022). SARS-CoV-2 Omicron Variant Neutralization after mRNA-1273 Booster Vaccination. N. Engl. J. Med. NEJMc2119912.

Peacock, T.P., Goldhill, D.H., Zhou, J., Baillon, L., Frise, R., Swann, O.C., Kugathasan, R., Penn, R., Brown, J.C., Sanchez-David, R.Y., et al. (2021). The furin cleavage site in the SARS-CoV-2 spike protein is required for transmission in ferrets. Nat. Microbiol. 6, 899-909.

Planas, D., Veyer, D., Baidaliuk, A., Staropoli, I., Guivel-Benhassine, F., Rajah, M.M., Planchais, C., Porrot, F., Robillard, N., Puech, J., et al. (2021). Reduced sensitivity of SARS-CoV-2 variant Delta to antibody neutralization. Nature 596, 276-280.

Planas, D., Saunders, N., Maes, P., Guivel-Benhassine, F., Planchais, C., Buchrieser, J., Bolland, W.-H., Porrot, F., Staropoli, I., Lemoine, F., et al. (2022). Considerable escape of SARS-CoV-2 Omicron to antibody neutralization. Nature 602, 671-675.

Puranik, A., Lenehan, P.J., Silvert, E., Niesen, M.J.M., Corchado-Garcia, J., O’Horo, J.C., Virk, A., Swift, M.D., Halamka, J., Badley, A.D., et al. (2021). Comparison of two highly-effective mRNA vaccines for COVID-19 during periods of Alpha and Delta variant prevalence. MedRxiv Prepr. Serv. Health Sci. 2021.08.06.21261707.

Radvak, P., Kwon, H.J., Kosikova, M., Ortega-Rodriguez, U., Xiang, R., Phue, J.-N., Shen, R.-F., Rozzelle, J., Kapoor, N., Rabara, T., et al. (2021). B.1.1.7 and B.1.351 variants are highly virulent in K18-ACE2 transgenic mice and show different pathogenic patterns from early SARS-CoV-2 strains. 2021.06.05.447221.

Schmidt, N.J., Dennis, J., and Lennette, E.H. (1976). Plaque reduction neutralization test for human cytomegalovirus based upon enhanced uptake of neutral red by virus-infected cells. J. Clin. Microbiol. 4, 61-66.

Shang, J., Wan, Y., Luo, C., Ye, G., Geng, Q., Auerbach, A., and Li, F. (2020a). Cell entry mechanisms of SARS-CoV-2. Proc. Natl. Acad. Sci. 117, 11727-11734.

Shang, J., Ye, G., Shi, K., Wan, Y., Luo, C., Aihara, H., Geng, Q., Auerbach, A., and Li, F. (2020b). Structural basis of receptor recognition by SARS-CoV-2. Nature 581, 221-224.

Suzuki, Y.J., and Gychka, S.G. (2021). SARS-CoV-2 Spike Protein Elicits Cell Signaling in Human Host Cells: Implications for Possible Consequences of COVID-19 Vaccines. Vaccines 9, 36.

Waltz, E. (2021). COVID vaccine makers brace for a variant worse than Delta. Nature 598, 552-553. 
Whittaker, G.R. (2021). SARS-CoV-2 spike and its adaptable furin cleavage site. Lancet Microbe 2, e488e489.

Winger, A., and Caspari, T. (2021). The Spike of Concern-The Novel Variants of SARS-CoV-2. Viruses 13, 1002.

Winkler, E.S., Bailey, A.L., Kafai, N.M., Nair, S., McCune, B.T., Yu, J., Fox, J.M., Chen, R.E., Earnest, J.T., Keeler, S.P., et al. (2020). SARS-CoV-2 infection of human ACE2-transgenic mice causes severe lung inflammation and impaired function. Nat. Immunol. 21, 1327-1335.

Wu, L., Zhou, L., Mo, M., Liu, T., Wu, C., Gong, C., Lu, K., Gong, L., Zhu, W., and Xu, Z. (2022). SARS-CoV-2 Omicron RBD shows weaker binding affinity than the currently dominant Delta variant to human ACE2. Signal Transduct. Target. Ther. 7, 1-3.

$\mathrm{Xu}$, Z., Liu, K., and Gao, G.F. (2022). Omicron variant of SARS-CoV-2 imposes a new challenge for the global public health. Biosaf. Health.

Zhao, X., Li, D., Ruan, W., Zhang, R., Zheng, A., Qiao, S., Zheng, X., Zhao, Y., Chen, Z., Dai, L., et al. (2021). Reduced sera neutralization to Omicron SARS-CoV-2 by both inactivated and protein subunit vaccines and the convalescents. 2021.12.16.472391. 


\section{Figure legends}

Figure 1. Antibody responses upon immunization with VOC-based vaccines in vivo. (A) Day 14 sera post booster was evaluated for antibody binding to recombinant Spike from designated VOCs by ELISA. (B) Day 14 sera post booster was evaluated for nAb responses against designated live virus by $50 \%$ plaque reduction neutralization test (PRNT). Plots: mean \pm SEM; $n=6$. EPT: endpoint titer.

Figure 2. Immunization with Furin mutant vaccine produces enhanced protection in vivo. (A) Schematic illustration of the design of the in vivo study. (B, C) The lung viral titer (B) and the body weight (C) was measured in mice challenged with live SARS-CoV-2 VOCs after full immunization. Plots: mean $\pm S E M ; n=6$. ****p<0.0001 one-way ANOVA.

Figure 3. Immunization with Omicron-specific vaccine produces robust protection against Omicron challenge. (A) Day 14 sera post $2^{\text {nd }}$ vaccine shot were evaluated for $n A b$ responses against Omicron from designated VOC vaccine by pseudovirus assay. (B, C) Day 14 sera post $2^{\text {nd }}$ Omicron-specific vaccine shot was evaluated for antibody binding by ELISA (B) and for nAb responses by PRNT (C). Bars: mean \pm SEM; $\mathrm{n}=6$; ${ }^{* * *} \mathrm{p}<0.001$ by Student's t-test (D) Design of the in vivo study for Omicron-specific vaccine booster (left panel) and the viral titer in the lung from mice challenged with live Omicron virus (right panel). Plots: mean \pm SEM; $n=6$. **** $p<0.0001$ by one-way ANOVA.

Figure 4. Delta RBD-Omicron immunization induces the potent and broadly spectrum neutralization activity against SARS-COV-2 variants. (A) The schematic drawing shows the design of the chimeric Delta RBD-Omicron mRNA. The animals were immunized with the mRNA twice. The Day 14 sera post boost were evaluated for binding antibodies specific to recombinant $S$ proteins from designated VOC vaccines by ELISA. Bars: mean \pm SEM; $n=6 ;{ }^{*} p<0.01$ by Student's t-test. (B) Day 14 sera post boost was evaluated for $n A b$ responses by pseudovirus assay. Plots: mean \pm SEM; $n=6$. EPT: endpoint titer.

Figure S1. Expression and cleavage of Furin-mutated mRNA. (A) Design of the furin mutant mRNA. (B) The flow cytometry showed moderate increase in the surface expression of spike after furin cleavage mutation. (C) Full-length S protein is the dominant species in transfected 293T cells after the removal of furin cleavage site. (D) The mutation at the furin cleavae site also lowered the level of free S1 in the conditioned medium of transfected 293T cells. (E, F) Furin-cleavage mutant mRNA elicits higher neutralization antibody titers than wild type mRNA, revealed by both ELISA (E) and PRNT (F) on day 14 post boost. TM: transmembrane domain. FL: full-length spike. Plots: mean \pm SEM; $n=6$. 
bioRxiv preprint doi: https://doi.org/10.1101/2022.03.04.483032; this version posted March 7, 2022. The copyright holder for this preprint (which was not certified by peer review) is the author/funder. All rights reserved. No reuse allowed without permission.

Figure S2. Expression of Omicron and Delta RBD- Omicron mRNA. The flow cytometry showed the surface expression of spike protein in transfected 293T cells stained with recombinant human ACE2 receptor. 
Figure 1

A

A
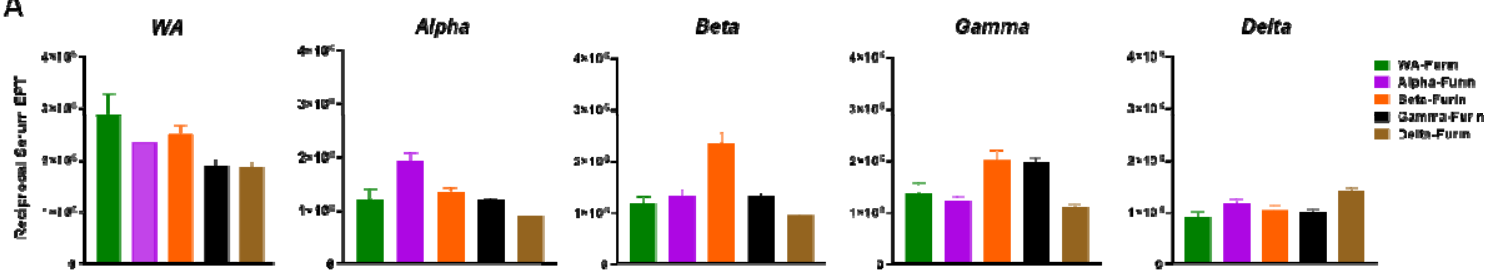

B

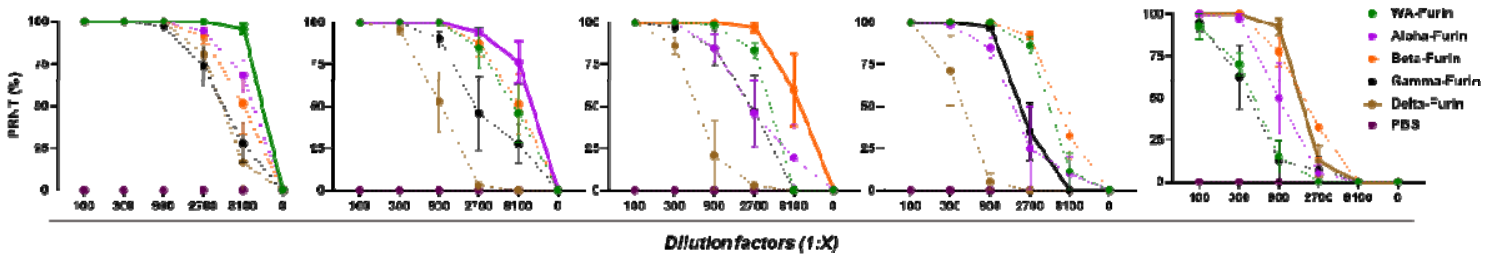


Figure 2

A

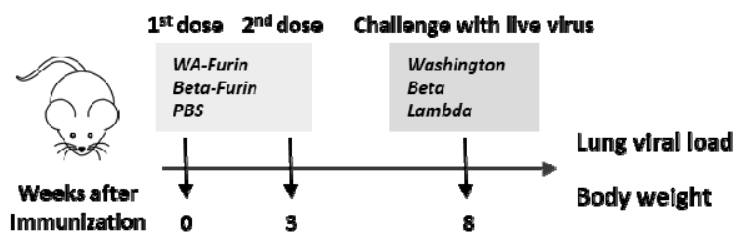

B
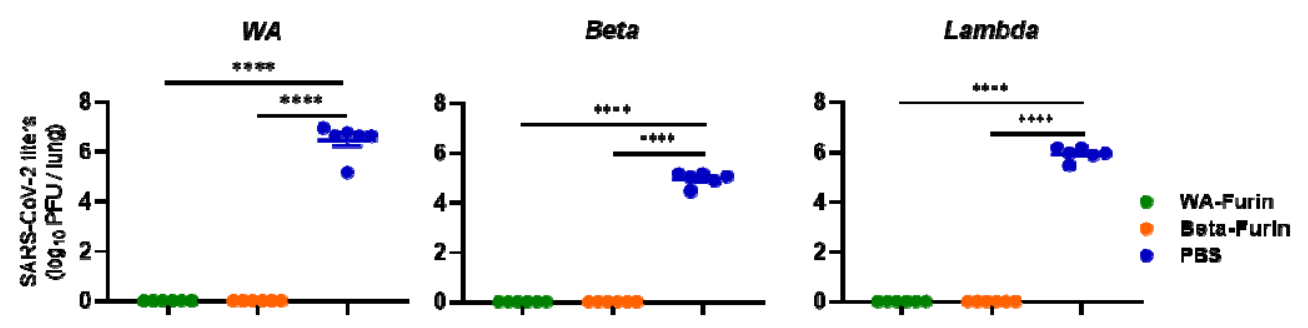

C
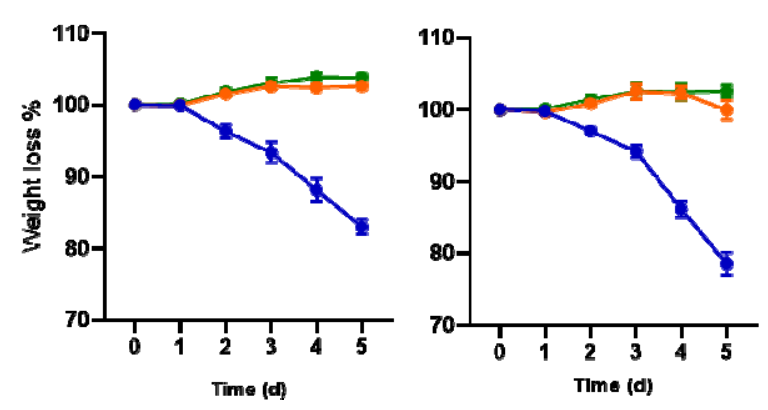
Figure 3
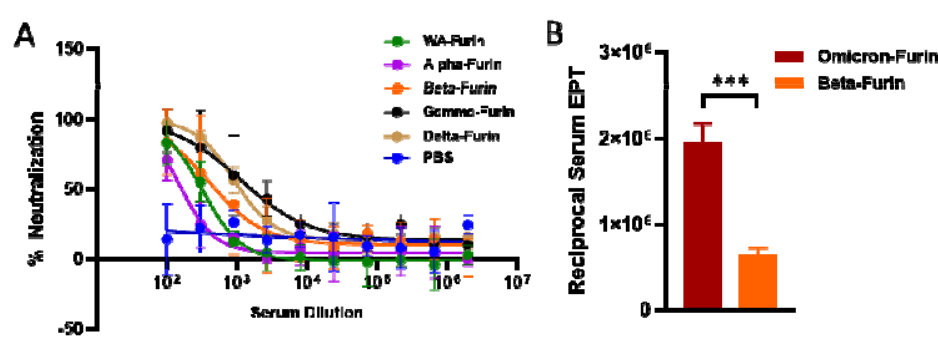

C

D
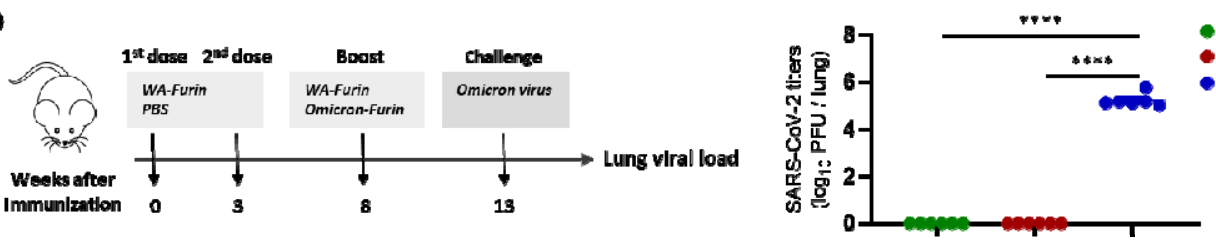

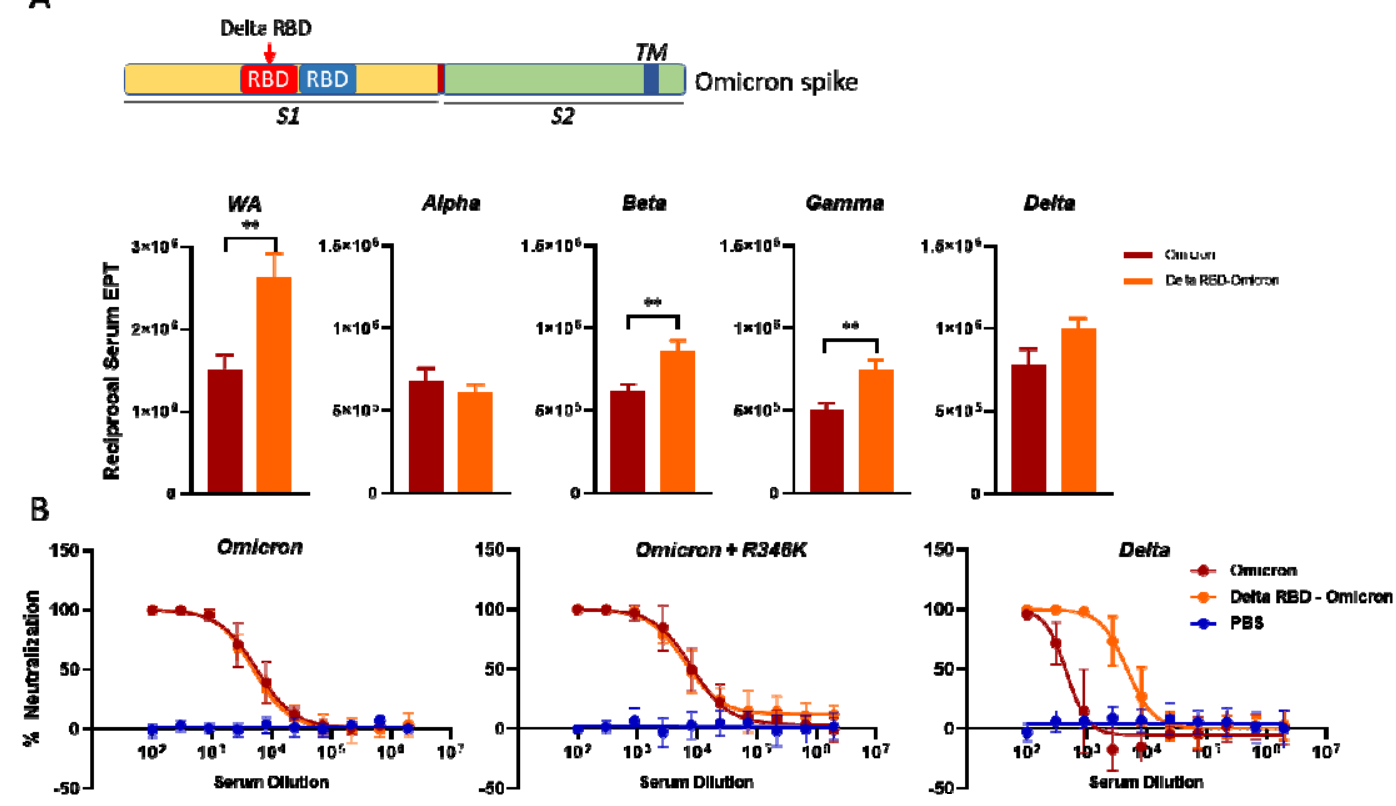
bioRxiv preprint doi: https://doi.org/10.1101/2022.03.04.483032; this version posted March 7, 2022. The copyright holder for this preprint (which was not certified by peer review) is the author/funder. All rights reserved. No reuse allowed without permission.

Supplementary Figure 1




bioRxiv preprint doi: https://doi.org/10.1101/2022.03.04.483032; this version posted March 7, 2022. The copyright holder for this preprint (which was not certified by peer review) is the author/funder. All rights reserved. No reuse allowed without permission.

Supplementary Figure 2
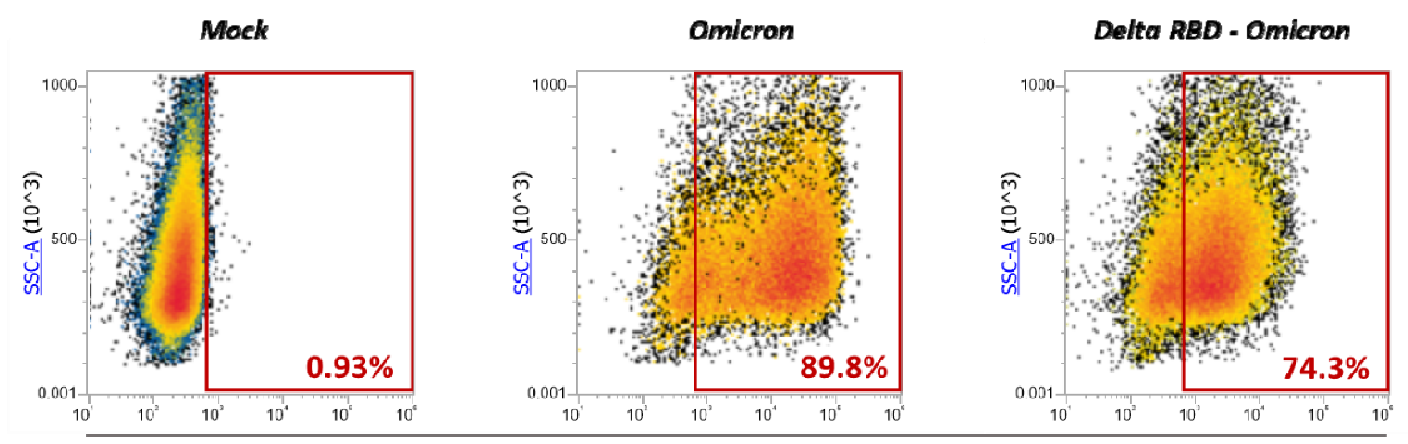

Binding to ACE2 